\title{
Editorial
}

\section{Minimizing our scientific footprints}

T ooking after our environment is a vexed business as it gets in the way of untrammelled freedom. We almost always need to stop doing things we are used to and spend more time and energy working out how to achieve our objectives with less impact both on habitats and species. And in the Antarctic this should surely be a priority.

Conservation and environmental impacts were not major issues for the architects of the Antarctic Treaty but SCAR was quick off the mark, recognizing the importance of implementing conservation measures in Antarctica before the Treaty had even met. The Agreed Measures of 1964 were the outcome of proposals from the science community and provided the basis for formalized conservation actions for decades after. However, all these were at the level of governance, proscribing areas and species for protection, but with no regard for monitoring of the impacts of the science and associated logistic activities themselves nor of personalizing the responsibilities to individuals. True, you had to get a permit to enter a protected area or to kill animals but there was little on how you should behave.

A major step forward was the development of a Code of Conduct for Experimentation on Animals, an initiative started by Knowles Kerry from Australia in the 1980s. Its original draft principles were watered down to the lowest common denominator as some scientists became concerned that their freedom of scientific action might be circumscribed, but it did at last provide a basic minimum standard and was later adopted into all the protected area management plans.

The development and implementation of the Protocol reflected a growing environmental awareness that more needed to be done to limit field impacts in Antarctica. How could we complain about possible tourism impacts if we were not adequately careful ourselves? US, Australian and New Zealand scientists drew up the Code of Conduct for the McMurdo Dry Valleys, the negotiations for which took several workshops and a real appreciation between disciplines of how they could work together. With some national operators now producing their own Codes of Conduct for field activities there was growing potential for confusion, especially where multinational groups were operating. Whose code should they follow and did it cover all the activities?

SCAR became engaged and initially the RiSC community produced their own Code to try and limit the introduction of alien species. But a composite code was needed, pulling together all the best practices and using a unified terminology. After extensive consultation the new Environmental Code of Conduct for Terrestrial Scientific Field Work in Antarctica was finally adopted by the delegates at the recent meeting in Moscow.

It is written in a personalized style and is meant to be included within the operational documents for each country. But have arrangements been made to translate it into all the national languages? COMNAP has commented on it but just how will it be included in the training programmes for all those going to Antarctica? And how will we know how effective it is?

There is also a new version of the Scientific Code of Conduct for Experiments on Animals under discussion. Many countries have now tightened up their legal rules for animal experiments to minimize suffering and limit deaths. Antarctica should not be an enclave free of these modern controls but as a continent for science it should be at the forefront of such activities. Antarctic scientists have a responsibility to conform to the highest rather than the lowest standards - so let us get both the new Codes into place as soon as possible.

DAVID W.H. WALTON 